\title{
TECHNOLOGICAL FORMS DEVELOPE IN DIFFERENT MORPHOLOGICAL FIELDS
}

\author{
Delia Garleanu, ${ }^{1, *}$, Delicia Arsene ${ }^{1}$, Gabriel Garleanu ${ }^{1}$, and Claudia Borda ${ }^{1}$ \\ ${ }^{1}$ UPB, IMST Faculty, TMS Department, 060042 Splaiul Independentei 313, Bucharest, Romania.
}

\begin{abstract}
The present work is takes in consideration the experimental study of the influence of some morphological fields regarding the development of the complex technological forms. The goal that we have set in this paper was to bring in new points of view on technology morphologies arising in technological processes, whose goal is to generate surfaces.The development of the technological shapes could be controlled and oriented from the quality and quantity point of view, by using the morphological fields.. We refer here in particular to complex forms, not the Euclidean upon which adequate studies have been done already. Among the illustrations of the experimental parameters of forming events, electrochemical deposit is regarded as a paradigm for theoretical studies of diffusion limited aggregation. In fact, by changing the concentration of metal ions, the cathodic potential and the morphological field you can explore different morphologies, such as: dense radial aggregates, dendridic patterns and fractal aggregates. This topic has been treated and shown in the paper. The box-counting algorithm was used in calculating the fractal dimension of the deposited agregates.
\end{abstract}

\section{Introduction}

In order to bring some extra objectivity in evaluating the shapes, we have done an experiment and study regarding the Copper dendrite growth, by electrolytic deposit in a CuSO4 solution, under the presence of some Euclidian morphological fields.

In the same time, we have done a selection of the resulted structures, that were considered relevant from the auto-similarity point of view, in order to attach fractal like interpretation mathematical procedure.

As in the field, virtually no research has been done, we were required to develop investigative techniques specific to consider estimators of complex shapes and their quantitative evaluation.

\section{Experimental context}

We tried to achieve, through the experiments we've carried out, some electrodeposited geometries, in wide variety, in order to be able to investigate, as well as possible, the clusters shape. That's why we used different experimental conditions. We present here under the parameters that we controlled in the process.

\subsection{Operation Stand}

Electrolysis cell is connected to the adjustable voltage source, a current transducer is interposed between the cathode and the source mass ( $2 \Omega$ resistor).
Electrolysis cell anode is attached via an electrical insulator, to the pan balance. An electronic opto-coupler device is attached to the balance and a shutter is fixed to its needle. Thus the change of mass is converted by the opto-coupler, by the deflection of the needle balance into an electrical signal through the amplifier for the optocoupler.

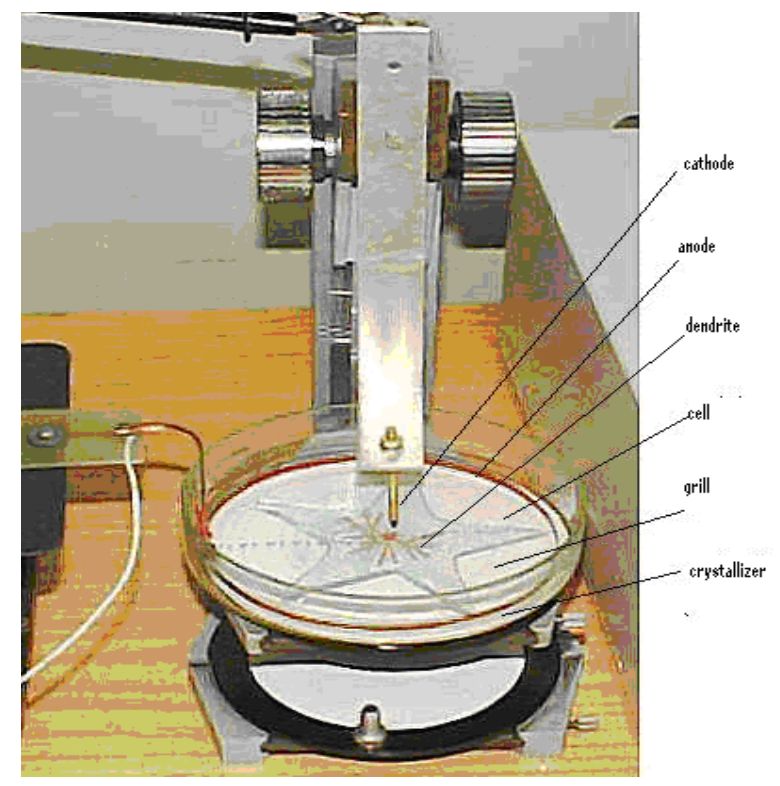

Fig. 1. Operation stand.

\footnotetext{
*Corresponding author: delia.garleanu@upb.ro
} 
Electrical signals for the effects of mass and current are sent to the data aquisition card from the computer, thus it can be viewed as built with LabVIEW as well as on an oscilloscope and a multimeter.

Further, in detail, is presented the electrolytic deposited cell.

It consists of:

- Crystallizer;

- $\mathrm{Cu}$ Anode ring;

- Cathode brass cylinder, $1.5 \mathrm{~mm}$ in diameter and 3mm length;

- CuSO4 solution;

To have the prospect of dendritic image formed by scanning, cathode is mounted on a glass plate with different geometric shapes.

Under crystallizer it was placed a concentric grid while tracking the development of the cluster radial electrodeposition.

\subsection{Introducing morphological fields}

The electrolyte type: Copper sulphite and the quality of the soluble anode, that was electrolytic copper.The thickness of the electrolyte layer: it was limited at 0,5-1 $\mathrm{mm}$ in order to prevent the growth of the electrodeposited cluster but only in $2 \mathrm{D}$ and not in $3 \mathrm{D}$ dimension.

The Copper sulphite solution's concentration: there have been carried out several procedures, with different concentrations of the solution, from $5 \%$ to $30 \%$. The $30 \%$ concentration was the saturation one. Within $5 \%$ to $10 \%$ concentration the process was very slow and the deposited material was insignificant. For the $15 \%$ concentrations the fractals' geometries were highly ramified but that was dependent on the current parameters, tension and intensity. For the higher concentrations, bigger than $15 \%$, the dendrite patterns are more dense, the ramification capacity decreases and the main growth directions are most visible ones.

The tension and intensity of the electric power: from this point of view there have been observed two cluster categories: those which have grown under constant tension and those which have grown under constant intensity.

The clusters that grown under constant tension presented ramified geometries, between the dendritically and fractal patterns, depending on the concentration of the electrolyte solution:

- for the concentrations between $15 \%$ and $20 \%$ there take place the ramified growth with self-scaling capacity.

- for the concentrations over $20 \%$ up to saturation, there begin to show up, step by step, dens morphologies, with faint or with no ramifications.

There have been used different tensions for the current but the most interesting results have been obtained for $\mathrm{U}=10 \mathrm{~V}$. The intensity varied from $0,18 \mathrm{~A}$ to 1A. The clusters that grew under constant intensity show a limitation of the growth diameter, after the cathode potential was decreased to $6 \mathrm{~V}$. From here on the experiment reveals that the deposited material grows up to the limits of the aggregate, extending its surface a lot, in the volume that the liquid level allows. In this case it was realised a 3D fractal aggregate that was limited at the cluster's margins.

The intensity of the current had the limits between $0,2 \mathrm{~A}$ and $0,8 \mathrm{~A}$, but the most spectacular results were obtained between $0,4 \mathrm{~A}$ and $0,6 \mathrm{~A}$; and this happened because for $0.4 \mathrm{~A}$ the growth was very slow and did not go over $30-35 \mathrm{~mm}$ diameter and for intensities bigger than $0,6 \mathrm{~A}$ we should have grown the tension over $15 \mathrm{~V}$ which would have conduct to boiling phenomena around the cathode.

We present in figure 2 some of the results of these experiments.

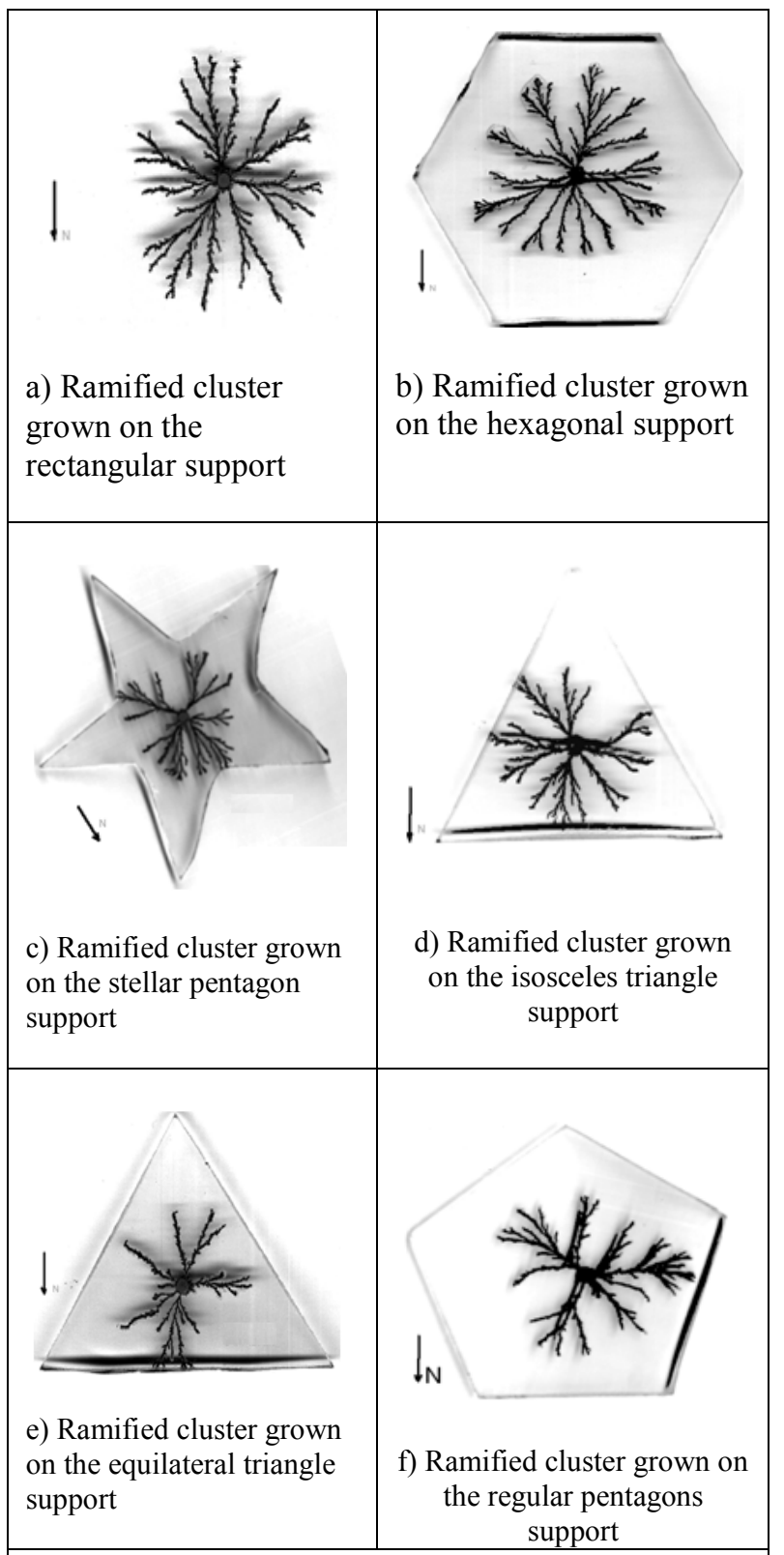

Fig. 2. Ramified clusters grown in some morphological fields in electrolyte solution with the weak concentrations (between $15 \%$ and $20 \%$ )

The geometry of the morphological fields: it was introduced through a glass support, on which we fixed the cathode and where the clusters were developing. 
In these conditions, the image capture, used for scanning, was eased a lot. There were used different shapes for the glasses supports, such as circles, with different diameters, regular pentagons, stellar pentagons, hexagons, triangle, square forms.

The influence on the morphological fields was more obvious for the circles, stellar pentagon and hexagon.

For the circle shape supports, the cluster had a constant growth speed and approximately equal on each brunch; all the brunches reached the same diameters in the same time. As for the stellar pentagon there have been observed a bigger growth in favour of those directions not oriented to the corners and even though the deposited material was made after radial patterns, these directions were more favourite.The clusters in the hexagonal supports showed regular geometries, with approximately equal angles between the main ramifications and similar growth, as number and angles, on each brunch. The other morphological fields did not have such a strong influence on the clusters geometry.

In the figure 2 we show the clusters with some geometries, grown in the constant intensity, that were obtained by electrodepositing: strongly ramified, with self-scaling capacity, weakly ramified or not ramified at all, radial.

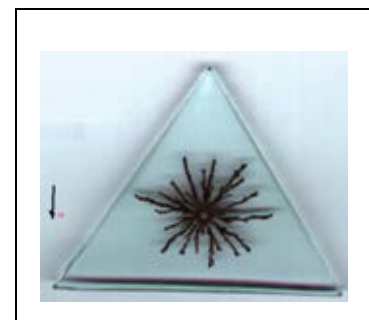

a) Unramified radial cluster grown in the weak concentration solution

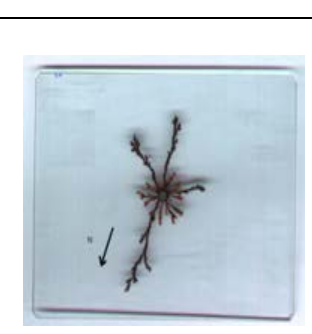

c) Radial cluster limited in diameter, grown in the weak concentration solution

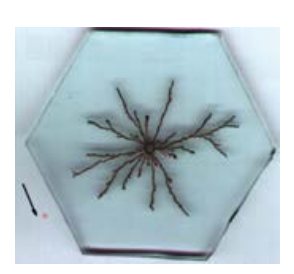

b) A little ramified cluster grown in the weak concentration solution concentration solution

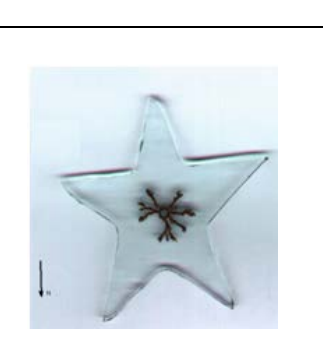

d) Radial cluster limited in diameter, grown in the weak concentration solution

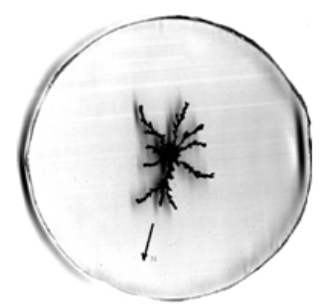

e) A little ramified cluster grown in the high concentration solution

Fig. 2. Clusters with some geometries, grown in the constant intensity.
There have been tried a certain positioning after the cardinal points and the moment of the day when the experiment took place but there was no connection between these parameters and the shape or size of the electro-deposited shape.

It is important to notice that the study of the self similar fractal aggregates shows that these morphologies are to be found as product of other processes and phenomena In this respect, there could be applied a fractal mathematical algorithm, that already exists and could be used in order to study these objects.

There is to be mentioned that the angles between the aggregates ramifications are pentagonal geometries with $36^{\circ}$ main values. As for the ramification value, this was level 3, as for an object is considered to be fractal if it is capable for scaling at least on 3 levels. So, in this study we didn't give any attention to the non-ramified geometries or to those weakly ramified and not even the radial ones, because none of them had self-similarity for more than 2 levels scaling.

\subsection{Generalized fractal dimensions}

In the context of this paper, the generalized fractal dimensions will be used to quantify the self similarity properties of numerical fractal aggregates and experimental ones.

When investigating scaling properties of fractal geometry aggregate measure $\mu$ is the mass of the object.

To define a square with a uniform grid $\varepsilon$, size (Fig. 4.1). Let I denote by $\mu_{I}$ proportion of the total mass of the object that is contained inside the box "and" for an " $i$ ": for an empty box $\varepsilon$ does not exist.

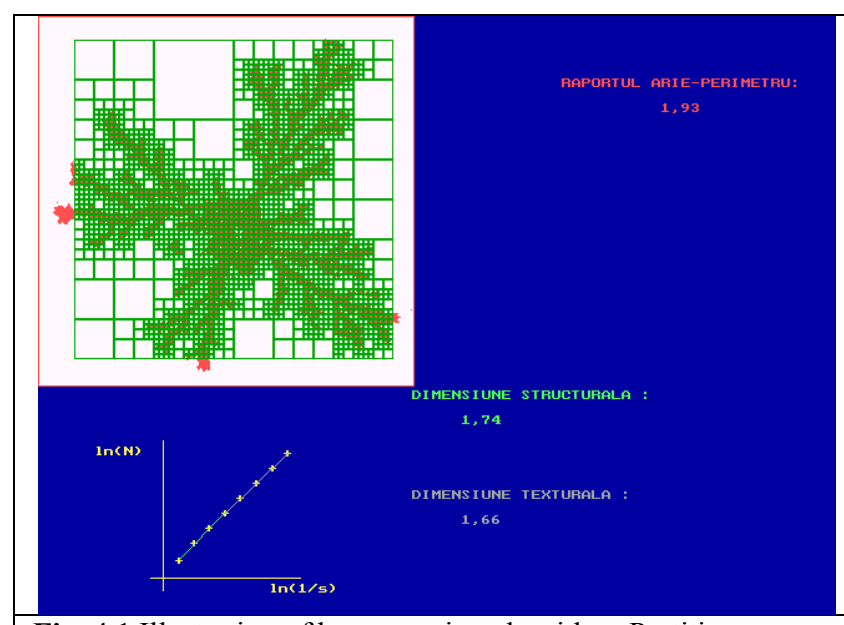

Fig. 4.1.Illustration of box-counting algorithm. Partition function $\mathrm{zq}(\varepsilon)$, is calculated using a grid of square boxes $\varepsilon$. side. $\mathrm{D}_{\mathrm{q}}$ dimensions are estimated by averaging the random positions on the grid in accordance with the unit.

As we know, one of the essential characteristics of fractal objects is that they manage to pack in a relatively small zone very high perimeters, in case of twodimensional structures, or in relatively small volumes large areas for 3D structures. If we look at 2D and to define the terms "relatively small" and "relatively large" we say that the Euclidean perimeter which delineate a 
surface area increases theoretically unlimited when it is a fractal object, depending on the unit size that measured it. So we can say that if:

$$
\begin{aligned}
& 1 \rightarrow 0 \text { then } \\
& \mathrm{p} \rightarrow \infty \text { and } \\
& \mathrm{A} \cong \mathrm{ct} .
\end{aligned}
$$

where: 1 is the length unit which made the perimeter measurement; $\mathrm{p}$ - the fractal object perimeter; $\mathrm{A}-$ the fractal object area.

These unlike the Euclidean figures where the perimeter size does not depend on the length unit of which the measurement is made. So we thought appropriate that in addition to other morphological investigation techniques presented to introduce the areaperimeter relation, we can give us a measure of the complexity of object edges, a measure of the packing degree; that's why we will call it, the wrapping dimension.

\subsection{The work algorithm}

We call the packing dimension the perimeter log slope graph function of log area length unit which we measure it.

Both the generated articles by iterative functions and for the natural objects (the $\mathrm{Cu}$ electrolytic depositions) is measured with perimeter different lengths. It plots log (Perimeter) / log (measurement unit) and the obtained graph slope is calculated by the interpolation function. This is the packing dimension. It characterizes the subject from the global point of view.

The fractal dimensions obtained for the deposited clusters varied between 0,8 and 1.98 as follows:

for simple Euclidean square shapes, circle and weak consonants of the solution the fractal dimensions were in the beginning of the size range

for Euclidean forms with multiple sides, the fractal dimensions were increased with the complexity of the geometry and the concentration of the solution

Any object is an information carrier and the higher the delimiter surface is, the greater the storage capacity of the information and the interfacing with the environment in which it is sunk.

Considering the above, we can introduce a controlled development of such information-bearing objects, through the variation of the morphological fields and the parameters that condition the formation of clusters.

Also, we have introduced an algorithm for calculating the packing size for the crossing into concrete of the complex structures characteristics.

The packaging size provides a clear relationship between the volume of the object and the surface that encompasses it.

The lower the volume and the larger the packaging surface, the greater the storage capacity and the more intense the exchange of information are..

\section{Conclusions}

The study of the self similar fractal agregates shows that these morphologies are to be found as product of other processes and phenomena there could be applied a fractal mathematical algorithm, that already exists and could be used in order to study different objects.

The development of the technological shapes could be controlled and oriented from the quality and quantity point of view, by using the morphological fields.

The introduction of some characteristic features of the surface/perimeter ratio contributes to the quantification of complex structures.

In this respect, technologies can be developed to produce objects with a high capacity for storing information in small volumes and to convey an important information flow.

We intend, in the future, to control the growth by using "growth morphological inhibitors" and "field concentrators" which should introduce morphological fields of different qualities.

\section{References}

1. D. Arsene, Contributions regarding the technological shapes stability and their interpretation through the fractal theory, Doctor Thesis, University Politechnica Bucharest, (1999)

2. G. Palla, P. Pollner and T.Vicsek, Rotated multifractal network generator, Journal of Statistical Mechanics: Theory and Experiment (2011).

3. Z. Kazim, The Hausdorff and box dimension of fractals with disjoint projections in two dimensions, Glasgow Math J 44:117-123 ( 2002).

4. Lapidus ML, van Frankenhuijsen M, Fractal geometry and Applications, part 1, 2 American Mathematical Society, Providence (2004).

5. Y. Heurteaux, Dimension of mearures; the probabilistic approach, Publ.Mat.51, 280-290, (2007).

6. Ben Avraham D., Havlin S., Diffusion and reactions in fractals and disordered systems, Cambridge University Press, Cambridge (2000)

7. Jaffard S, Meyer Y, Ryan RD, Wavelets: Tools for science and technology, SIAM, Philadelphia (2001).

8. Kenneth Falconer, Fractal Geometry: Mathematical Foundations and Applications, 3rd Edition, , ISBN 978-1-119-94239-9, 398pg, Jan (2014).

9. D-J Feng, KS Lau, Multifractal formalism for selfsimilar measures with weak separation condition, J. Math.Pures Appl., 92, (2009).

10. D. Moise, B. Bogdan, D, Druta, Aldoritmi, numere si fractali, Ed Printech, (2007).

11. A. Bunde, S. Halvin, Fractals and disordered systems, Springer Science, (2012). 